\title{
La visión Skinneriana sobre las alucinaciones. Vigencia y revisión
}

\author{
José M. García Montes ${ }^{*}$ y Marino Pérez Álvarez ${ }^{2}$ \\ ${ }^{1}$ Universidad de Almería ${ }^{2}$ Universidad de Oviedo \\ (Recibido 12 Marzo 2011; Aceptado 24 Abril 2011)
}

RESUMEN: El presente trabajo expone brevemente la visión que B.F. Skinner mantenía sobre las alucinaciones como fenómenos psicológicos, y defiende su vigencia y relevancia hoy en día. Para ello se parte de la concepción skinneriana sobre las conductas de percibir, imaginar y soñar. Se insiste en que, según el genial psicólogo americano, las diferencias entre la percepción por un lado y la imaginación o el sueño por otro, se centran en qué tipo de variables controlan el comportamiento en cuestión. Así el percibir estaría controlado principalmente por la estimulación circundante a la persona, mientras que la imaginación o el sueño lo estarían por variables que se hallan "debajo de la piel" del sujeto y, por lo tanto, son privadas e inaccesibles a la comunidad verbal de forma directa. Partiendo de ello, Skinner viene a entender las alucinaciones como conductas perceptivas que se realizan en ausencia del estímulo percibido, similares a las conductas de imaginar o soñar, pero que la persona, por diversas razones, no reconoce como controladas fundamentalmente por la estimulación privada. Finalmente se realizan algunas consideraciones críticas que atañen a la falta en Skinner de una teoría radical sobre la persona.

Palabras clave: alucinaciones, conductismo, imaginación, discriminación del propio comportamiento.

\section{Skinner's view on hallucinations. Validity and revision}

\begin{abstract}
This work outlines the vision that B.F. Skinner held on hallucinations as psychological phenomena, and defends its validity and relevance nowadays. Our thesis starts with the Skinner's conception on the behaviors of perceiving, imagining and dreaming. It is highlighted that, in Skinner's point of view, the differences between perception on one hand and imagination or dreaming in the other, focus on what kind of variables control the behavior in question. Thus, the perceptive behavior would be controlled primarily by stimulus surrounding the person, while the imagination or dreaming would be by variables that are "under the skin" of the subject and, therefore, are private and directly inaccessible to the verbal community. On this basis, Skinner comes to an understanding of hallucinations as perceptual behaviors that occur in the absence of a perceived stimulus, similar to the behavior of imagining or dreaming, but, when hallucinating, the person, for various reasons, does not recognize his / her behaviour as controlled mainly by private stimulation. Finally we made some critical remarks regarding the lack in Skinner's psychology of a radical theory on the human person.
\end{abstract}

Keywords: hallucinations, behaviorism, imagination, own behaviour discrimination. 


\section{INTRODUCCIÓN}

Aun cuando B. F. Skinner (1904 - 1990) no desarrolló in extenso una posición sobre las alucinaciones, sí que aportó en obras diversas consideraciones fundamentales que todavía hoy, medio siglo después, consideramos vigentes. En el presente trabajo se resume de la forma más sistemática posible el meollo de la posición skinneriana para, a continuación, añadir algunas valoraciones propias. Para ello, se parte de la concepción conductista sobre lo que es la percepción en cuanto la alucinación viene definida en los sistemas psicopatológicos al uso en un sentido negativo como una "percepción sensorial que tiene el convincente sentido de la realidad de una percepción real, pero que ocurre sin estimulación externa del órgano sensorial implicado" (American Psychiatric Association, 2003, p. 914). Al comenzar así, desde la percepción, no intentamos situar a Skinner al lado de las teorías que entienden las alucinaciones como fenómenos que se imponen al sujeto; más bien pretendemos resituar la percepción como comportamiento, algo sobre lo que el propio Skinner insistió en repetidas ocasiones. Si percibir es comportarse, alucinar también lo es y, en consecuencia, las alucinaciones son conducta operante (por más que muchas veces sus condiciones de aparición, de mantenimiento y de cambio no sean detectables con un análisis superficial de la situación).

\section{PERCIBIR, IMAGINAR Y SOÑAR DESDE UNA PERSPECTIVA SKINNERIANA}

Para Skinner, como no podía ser de otra manera, percibir es actuar. En contra de una opinión común en su época, para Skinner los datos sensoriales no son hechos impuestos, sino que son construcciones que devienen de la interacción entre un ser y su entorno. Por ende, percibir, como cualquier otra conducta, tiene una funcionalidad operatoria. Para decirlo con palabras del propio Skinner:

... en cierto sentido la percepción tiene un propósito o una intención. La persona no es un espectador indiferente que absorbe el mundo como una esponja [...] Nosotros no somos simplemente "captadores" del mundo que nos rodea; respondemos a él de maneras peculiares debidas a lo que ha sucedido cuando hemos estado en contacto con él (Skinner, 1994, p. 75).

Dicho de otra forma: el percibir, como conducta que es, está sometido a toda una serie de circunstancias (de contingencias, si se prefiere) de las cuales es función. Una de estas circunstancias sería el nivel de estimulación presente en un momento dado; otra, el nivel de privación de quien realiza la conducta de percibir. Ambas condiciones vienen a interactuar de tal forma que si una de ellas fuera débil, la otra jugaría un mayor papel en el control de la conducta de percibir. Así como sigue diciendo Skinner: 
... cuando un estímulo es débil o vago, hay otras condiciones que afectan la probabilidad de que una persona vea una cosa de determinada manera. El enamorado cree ver a su amada en medio de una multitud solamente cuando el estímulo visual es fugaz $\mathrm{u}$ oscuro [...] Las personas que responden vigorosamente a los ladrones o a los ratones oyen como si fuera hecho por ladrones o ratones cualquier ruido leve en la noche. El nivel de privación produce una diferencia: Erróneamente "oímos sonar el teléfono" cuando esperamos una llamada importante, y la persona privada sexualmente ve falos o vaginas en objetos que tienen muy poca semejanza con tales órganos (Skinner, 1994, p. 75-76).

Ya antes, en Conducta Verbal, Skinner había insistido sobre la importancia que cobran otras variables, generalmente inaccesibles a la comunidad verbal, cuando la estimulación presente en un determinado momento es débil:

Es común -señala Skinner- que no se oiga bien un estímulo verbal; pero si el estímulo ecoico es débil, no se sigue que la respuesta sea indeterminada. Otras variables simplemente tienen mayor probabilidad de ser efectivas como fuentes suplementarias de fuerza. Cuando tales variables son aparentes, decimos que el error de audición es "revelador" en sentido freudiano. Así, si alguien oye mencionar su nombre en una conversación ruidosa cuando, de hecho, no existe ningún estímulo verbal correspondiente, podemos buscar qué otra variable puede ser la responsable de la tendencia del individuo a oír mencionar su nombre. El padre que está orgulloso de su hijo oye más palabras en los balbuceos infantiles de su hijo que las que oye el vecino escéptico (Skinner, 2001, pp. 275-276).

Por lo tanto, de una u otra forma, entiende Skinner que la percepción está cosida con la imaginación. Las circunstancias que determinan la conducta de percibir no son sólo "externas", no estarían sólo "fuera de la piel"; sino que "el mundo dentro de la piel" es también fundamental a la hora de percibir, de idéntica forma a lo que ocurre con la imaginación.

Ahora bien, ¿qué es imaginar? De nuevo no cabe más que una respuesta: Imaginar es comportarse. Está claro que el que imagina (o quien sueña) no se comporta de forma abierta, pero sí lo hace de forma encubierta. Así Skinner, al tratar el tema de la imaginación, vuelve a insistir sobre su naturaleza de comportamiento operante:

... lo que se debe decir es que si recibimos refuerzo viendo Venecia, es probable que emitamos ese comportamiento (es decir, el comportamiento de ver Venecia) aunque en el contexto inmediato haya muy pocas cosas que guarden semejanza con la ciudad [...] En el sueño se 
encuentra un ejemplo más claro de "ver" en ausencia de la cosa vista. La estimulación actual reduce su control al mínimo y es cuando la historia y los estados resultantes de privación y emoción de la persona logran su oportunidad. Freud destacó la importancia de los deseos y los temores plausiblemente inferidos de los sueños, pero, desafortunadamente, se hizo responsable del énfasis en la distinción entre ver y lo que se ve. El soñador entraba en el sueño; montaba el sueño como el productor de teatro monta una obra y luego tomaba asiento entre el auditorio y lo observaba. Pero el sueño es un comportamiento perceptual, y la diferencia entre el comportamiento cuando se está despierto y cuando se está dormido, sea dentro o fuera de un contexto pertinente, es simplemente una diferencia en las condiciones que lo controlan. Parece que los movimientos rápidos de los ojos durante el sueño confirman esta interpretación. Cuando sueñan más activamente las personas mueven los ojos como si estuvieran observando una presentación visual (parece que también los músculos del oído medio se mueven durante los sueños e involucran percepciones auditivas). (Skinner, 1994, pp. 82-83, cursiva del autor ).

En resumidas cuentas, según Skinner

El meollo de la posición conductista sobre la experiencia consciente puede resumirse de este modo: El ver no implica algo visto. Adquirimos la conducta de ver bajo la estimulación de objetos reales, pero puedo ocurrir en la ausencia de estos objetos, bajo el control de otras variables. (Por lo que hace al mundo dentro de la piel, siempre ocurre en ausencia de tales objetos). También adquirimos la conducta de ver que estamos viendo cuando vemos objetos reales, pero también puede ocurrir en su ausencia (Skinner, 1982, p. 213).

Pues bien, esta diferenciación entre la conducta de percibir y la de reparar en que se está realizando la conducta de percibir será esencial a la hora de fundamentar una posición cabal sobre las alucinaciones desde el punto de vista skinneriano, como veremos en el siguiente apartado.

\section{LAS ALUCINACIONES COMO CONDUCTAS ENAJENADAS}

En efecto, a partir de lo dicho se nos aparece la alucinación como una cuestión de enajenación o alienación; esto es, una atribución al medio de un comportamiento que alguien realiza $\mathrm{y}$, sin embargo, no lo reconoce como propio. Para decirlo en términos más estrictamente conductuales: la persona que alucina no es consciente de las contingencias que controlan su conducta de oir-en-ausenciade-la-cosa-oída y de las cuales es función (privación, estados emocionales, historia, etc.). Cuando el sujeto repara en que se está comportando, aún en ausencia 
de la cosa "percibida", no cabría hablar strictu sensu de "alucinación"; sino de "pseudoalucinación", término que la jerga psiquiátrica reserva a este fenómeno de "alucinación" con conciencia de que se está alucinando. Por lo tanto, las diferencias entre los términos "percepción", "ilusión", "pseudoalucinación” y "alucinación" se nos presentan estrictamente contextuales. La percepción de un determinado objeto se suele dar en presencia de ese objeto que es, de forma principal, el estímulo que controla la conducta perceptiva. Cuando la percepción se produce ante claves ambientales ambiguas, que son las que controlan la conducta aun en ausencia del objeto percibido, trataríamos con una "ilusión". Cuando estas claves ambientales son inexistentes o mínimas, trataríamos con alucinaciones o pseudoalucinaciones en función de si la persona no repara (o sí lo hace en el caso de la pseudoalucinación) en su propio comportamiento de percibir-enausencia-de-la-cosa-percibida.

Cabría entonces preguntarse cuáles son las causas por las que un comportamiento como es el percibir-en-ausencia-de-la-cosa-percibida puede llegar a convertirse en una conducta enajenada, de tal forma que la persona no reconozca las circunstancias que controlan su comportamiento, habiendo realizado perfectamente esta discriminación en otros momentos anteriores.

En primer lugar, sería posible que la persona no reconozca las variables que de hecho están controlando su conducta de percibir-en-ausencia-de-la-cosa-percibida al ser poco habituales y, por decirlo así, excepcionales. Así, p. ej., en estados de fuerte privación o de gran ansiedad es posible que ciertos estímulos hasta entonces irrelevantes en el control funcional de un comportamiento perceptivo pasen a ser importantes $y$, así, controlen el comportamiento aún en ausencia de la cosa percibida. Algo parecido le ocurrió a Charlot en "La Quimera del Oro" cuando, atormentado por el hambre, ve a su compañero convertido en un pollo. La persona no estaría acostumbrada a que su conducta de percibir-en-ausenciade-la-cosa-percibida esté bajo el control de estas circunstancias tan intensas y, de esta forma, es posible que el comportamiento realizado se atribuya a la presencia de la cosa percibida en detrimento de los estados privados intentos que, de facto, han controlado el comportamiento.

En segundo lugar también es posible que, aunque se tenga contacto con las circunstancias que controlan un comportamiento, éstas resulten ser castigos verbales para la persona o, dicho de forma freudiana, resultaran inadmisibles al super-ego. La "censura", en tal caso, iría por la vía de la negación y la proyección. Y es que, como ya señalara Skinner:

Cuando no se ha presentado la retroalimentación de la conducta verbal en el momento de su emisión y cuando el hablante o escritor se encuentra con las evidencias de dicha conducta, es probable que la atribuya a otra persona. El hablante no sólo no recuerda haberla producido, sino que el material sin corregir puede ser tan extraño u objetable que le será irreconocible (Skinner, 2001, p. 418).

Sin duda esta enajenación o extrañamiento no sólo es propio de las alucinaciones, sino que también se puede presentar en otros síntomas psicóticos, como los delirios de control o inserción del pensamiento. Sin necesidad de llegar a 
fenómenos catalogados como psicopatológicos, Skinner señala la relevancia que tiene la conducta verbal "automática" (la que se realiza sin reparar en que se está haciendo) e indica, a modo de ejemplo, el caso de la escritura automática:

Un estudiante que había tenido éxito en un pequeño colegio universitario, en contacto estrecho con sus compañeros de facultad, ingresó en una escuela universitaria de graduados; en ella encontró que las cosas eran mucho más difíciles y que los profesores eran por completo indiferentes a sus problemas. Al asistir a una conferencia particularmente difícil, el estudiante dejó de tomar notas y, en forma muy lenta, llenó la página de su cuaderno con gran cantidad de garabatos infantiles que decían: No puedo seguir, por favor, quiero regresar. Al final de la conferencia miró la página y exclamó: ¡Mira lo que hice! Por supuesto, lo que había escrito de forma automática habría sido rechazado antes de emitido bajo condiciones "normales" (Skinner, 2001, pp. 416-417).

Con ello, Skinner sitúa las alucinaciones en una lógica propia de las conductas enajenadas; esto es, de conductas que por un lado se hacen y el hacerlas conlleva una cierta consecuencia positiva (alivio de la tensión, etc.); pero que, al tiempo, su autoría supone algún tipo de castigo o consecuencia negativa (autoreproches, etc.).

Desde luego este tipo de conflictos, donde hacer algo es a la vez reforzado y castigado (Quiroga, 1999), se da con frecuencia en personas que atienden a consultas psicológicas y aún se podría decir que en cualquier persona que atraviese una vida mínimamente compleja. Por ello la resolución de los conflictos, aceptándose uno a sí mismo y al mundo como es, al tiempo que encaminando la vida hacia los logros que se desean, se ha propuesto como una terapia innovadora para pacientes con síntomas psicóticos y, especialmente, alucinaciones auditivas (Carmona, Cangas, García y Pedro, 2009; García-Montes y Pérez-Álvarez, 2001).

\section{REVISANDO A SKINNER}

A la hora de revisar las aportaciones de Skinner al entendimiento de las alucinaciones nos parece oportuno comenzar señalando la relevancia de sus análisis. A nuestro juicio cualquier autor que en nuestros días hable de las alucinaciones sin haber entendido la posición skinneriana queda automáticamente desautorizado. Skinner parte de una posición constructivista sobre la percepción, resalta el entretejido diario que se da entre nuestro comportamiento perceptivo y el imaginativo, subraya la relevancia de las variables motivacionales en la conducta de percibir-en-ausencia-de-la-cosa-percibida, pone de manifiesto el peso fundamental que la comunidad verbal (la cultura) juega para que un comportamiento perceptivo "alucinatorio" sea atribuido por una persona en concreto a las condiciones privadas que lo generaron o bien sea entendido como una percepción "pú- 
blica" o "real", etc. Sin haber dedicado su obra al estudio de la psicopatología, y sin haber sido tampoco excesivamente sistemático en sus aproximaciones al tema, Skinner ha sentado las bases de un entendimiento novedoso y cabal de las alucinaciones. Contrasta ello, según nuestro criterio, con la obra de otros autores, dedicados en exclusiva al estudio de la psicopatología y perdidos entre teorías de la mente, módulos neurocognitivos y demás sofisticaciones pseudo-explicativas.

En este sentido, pocas son las críticas que pueden hacerse a sus consideraciones. Si acaso, le faltaría a Skinner, a nuestro entender, una concepción molar de la persona humana. Aunque esto puede observarse en diversos aspectos de su teoría, es especialmente llamativo en los ejemplos que ofrece a propósito de sus explicaciones: el enamorado, quien responde de forma excesivamente alarmista ante los ratones, la persona que le gusta Venecia, etc. Todos ellos son, sin lugar a dudas, ejemplos muy ilustrativos de aquello que se está exponiendo; pero, a la vez, habría que señalar que son demasiado simples. Quien alucina de forma sistemática se encuentra alterado de una forma compleja y global. No se trata sencillamente de que la comunidad verbal castigue ciertos comportamiento, aun realizados de forma encubierta, y que, por consiguiente, la persona no los reconozca como propios. La persona que alucina suele encontrarse en posiciones sociales globalmente alteradas, suele afrontar conflictos, retos, rechazos, anhelos. En efecto, más allá de un comportamiento en concreto, cualquier ser humano ocupa una posición en un entramado histórico-social que le da contextos para formarse o para deshacerse. Se echa en falta en los análisis skinnerianos alguna referencia a este tipo de situaciones que, a nuestro parecer, tan fundamentales son para entender el comportamiento humano. Es verdad que Skinner abordó en diversas obras la influencia que determinadas condiciones sociales tienen sobre el comportamiento humano y que incluso ensayó en Walden 2 una sociedad "científicamente dirigida". Pero tales análisis, aun siendo relevantes, pierden de vista, a nuestro parecer, el aspecto fundamental: que persona y sociedad se determinan recíprocamente y que, por ende, no es suficiente establecer las condiciones sociales "óptimas". Si se habla ya de que unas condiciones en concreto son "óptimas" es porque previamente se tiene, si quiera de forma inconsciente, una concepción de qué es la persona humana. La tarea pendiente de la psicología, lindando entonces con la antropología, la sociología y la historia, sería, pues, la elaboración de esa teoría de la persona humana (vid., p.ej., Pérez-Álvarez \& García-Montes, 2004, 2006), tan cara a la cultura occidental y tan ajena en otras.

\section{AGRADECIMIENTOS}

Este trabajo ha sido realizado en el marco del proyecto de investigación SEJ2005-00455/PSIC, concedido por el Ministerio de Ciencia y Tecnología del Gobierno de España. 


\section{REFERENCIAS}

American Psychiatric Association (2003). Manual Diagnóstico y Estadístico de los Trastornos Mentales (DSM IV-TR). Barcelona: Masson.

Carmona, J.A., Cangas, A.J., García Montes, J.M., y Pedro, S. (2009). Terapia de aceitação e compromisso aplicada aos síntomas Psicóticos. Inuaf Studia, 14, 140-149.

García Montes, J.M. \& Pérez Álvarez, M. (2001). ACT como tratamiento de síntomas psicóticos. El caso de las alucinaciones auditivas. Análisis y Modificación de Conducta, 27(112), 455-472

Pérez Álvarez, M. \& García Montes, J. M. (2004). Personality as a work of art. New Ideas in Psychology, 22, 157-173.

Pérez-Álvarez, M. \& García-Montes, J. M. (2006). Person, behaviour and contingencies (an aesthetic view of behaviourism). International Journal of Psychology, 41, 449-461.

Quiroga, E. (1999). Tipología sistemática de la conducta. Psicothema, 11(1), 137-149.

Skinner, B.F. (1982). Contingencias de reforzamiento. Un análisis teórico. México: Trillas (Orig. 1969).

Skinner, B.F. (1994). Sobre el conductismo. Barcelona: Planeta-De Agostini. (Orig. 1974).

Skinner, B.F. (2001). Conducta verbal. México: Trillas (Orig. 1957). 